\title{
Some fixed point results for non-decreasing and mixed monotone mappings with auxiliary functions
}

\author{
Shuang Wang ${ }^{1 *}$, Arslan Hojat Ansari ${ }^{2}$ and Sumit Chandok ${ }^{3}$
}

\author{
"Correspondence: \\ wangshuang19841119@163.com \\ 'School of Mathematical Sciences, \\ Yancheng Teachers University, \\ Yancheng, Jiangsu 224051, \\ P.R. China \\ Full list of author information is \\ available at the end of the article
}

\begin{abstract}
The purpose of this paper is to provide sufficient conditions for the existence and uniqueness of fixed points for non-decreasing and mixed monotone mappings with auxiliary functions in the framework of metric space endowed with a partial order. As applications of our results we obtain several interesting corollaries and fixed point theorems in the underlying spaces. In order to illustrate our results, we provide two examples in which other theorems from the literature cannot be applied. In addition, the equivalence property between unidimensional and multidimensional fixed point theorems is investigated. We also present some applications to the existence of solutions of integral and differential equations.
\end{abstract}

MSC: $47 \mathrm{H} 10 ; 54 \mathrm{H} 25$

Keywords: non-decreasing mapping; mixed monotone mapping; fixed point; partially ordered metric space

\section{Introduction}

One of the newest branches of fixed point theory is devoted to the study of coupled fixed points, introduced by Guo and Lakshmikantham [1] in 1987. Thereafter, Gnana Bhaskar and Lakshmikantham [2] introduced the concept of the mixed monotone property for contractive operators in two variables in the setting of a partially ordered metric space, and they established some coupled fixed point theorems. Their results were extended and generalized by several authors in the last few years; see [3-21] and the references cited therein. Recently, Roldán et al. [22] introduced the notion of coincidence point between mappings in any number of variables, and several special extended notions of so-called coupled, tripled, quadrupled, and multidimensional fixed/coincidence points appeared in the literature; see, for example, $[2,3,13,23]$, respectively.

Harjani and Sadarangani [24] investigated some unidimensional fixed point theorems for generalized contractions in complete partially ordered metric spaces and applications to ordinary differential equations. In [23] and [25] the authors obtained some multidimensional fixed point theorems for mixed monotone mappings, which extended the corresponding coupled, tripled, and quadrupled fixed point results appearing in the literature. In 2014, Wang [26] obtained some multidimensional fixed point theorems for isotone mappings and extended some of the results in coupled, tripled, quadrupled and multidi-

(c) 2015 Wang et al. This article is distributed under the terms of the Creative Commons Attribution 4.0 International License (http://creativecommons.org/licenses/by/4.0/), which permits unrestricted use, distribution, and reproduction in any medium, provided you give appropriate credit to the original author(s) and the source, provide a link to the Creative Commons license, and indicate if changes were made. 
mensional fixed/coincidence points for mixed monotone and non-decreasing mappings in the framework of complete partially ordered metric spaces. She also gave a simple and unified approach to coupled, tripled, quadrupled, and multidimensional fixed point theorems for mixed monotone mappings.

Motivated and inspired by the results of [22-26], we establish some fixed point theorems for non-decreasing and mixed monotone mappings with auxiliary functions in a complete partially ordered metric space. The auxiliary functions used in the paper are more general than the gauge functions appearing in the literature. Our results improve and generalize the well-known results of Harjani and Sadarangani [24] and Wang [26]. By using the theorems, we also obtain several interesting corollaries and fixed point theorems in the underlying spaces. In order to illustrate our results, we provide two examples in which the theorems of [24] cannot be applied. In addition, the equivalence property between unidimensional and multidimensional fixed point theorems is investigated. Also as applications of our results, we provide two examples for the solution of integral and differential equations.

\section{Basic concepts}

In order to fix the framework needed to state our main results, we recall the following notions.

For simplicity, we denote from now on $\underbrace{X \times X \cdots X \times X}_{k}$ by $X^{k}$, where $k \in \mathbb{N}$ and $X$ is a non-empty set. If elements $x, y$ of a partially ordered set $(X, \leq)$ are comparable (i.e., $x \leq y$ or $y \leq x$ holds), we write $x \asymp y$. Let $\{A, B\}$ be a partition of the set $\Lambda_{k}=$ $\{1,2, \ldots, k\}$, that is, $A \cup B=\Lambda_{k}$ and $A \cap B=\emptyset, \Omega_{A, B}=\left\{\sigma: \Lambda_{k} \rightarrow \Lambda_{k}: \sigma(A) \subseteq A\right.$ and $\sigma(B) \subseteq$ $B\}$ and $\Omega_{A, B}^{\prime}=\left\{\sigma: \Lambda_{k} \rightarrow \Lambda_{k}: \sigma(A) \subseteq B\right.$ and $\left.\sigma(B) \subseteq A\right\}$. Henceforth, let $\sigma_{1}, \sigma_{2}, \ldots, \sigma_{k}$ be $k$ mappings from $\Lambda_{k}$ into itself, and $\Upsilon$ be the $k$-tuple $\left(\sigma_{1}, \sigma_{2}, \ldots, \sigma_{k}\right)$. For brevity, $\left(y_{1}, y_{2}, \ldots, y_{k}\right),\left(v_{1}, v_{2}, \ldots, v_{k}\right),\left(y_{1}^{n}, y_{2}^{n}, \ldots, y_{k}^{n}\right)$ and $\left(x_{0}^{1}, x_{0}^{2}, \ldots, x_{0}^{k}\right)$ will be denoted by $Y, V, Y_{n}$ and $X_{0}$, respectively.

Let $(X, \leq)$ be a partially ordered set and $d$ be a metric on $X$. We use the next notation from [22]:

$$
x \leq_{i} y \Leftrightarrow \begin{cases}x \leq y, & \text { if } i \in A, \\ x \geq y, & \text { if } i \in B .\end{cases}
$$

The product space $X^{k}$ is endowed with the following natural partial order: for $Y, V \in X^{k}$,

$$
Y \preceq V \quad \Leftrightarrow \quad y_{i} \leq_{i} v_{i}, \quad \forall i \in \Lambda_{k} .
$$

Obviously, $\left(X^{k}, \preceq\right)$ is a partially ordered set. The mapping $\rho_{k}: X^{k} \times X^{k} \rightarrow[0, \infty)$, defined by

$$
\rho_{k}(Y, V)=\frac{1}{k}\left[d\left(y_{1}, v_{1}\right)+d\left(y_{2}, v_{2}\right)+\cdots+d\left(y_{k}, v_{k}\right)\right],
$$

where $Y, V \in X^{k}$, is a metric on $X^{k}$. It is easy to see that, for $Y_{n}, Y \in X^{k}$,

$$
\rho_{k}\left(Y_{n}, Y\right) \rightarrow 0 \quad(\text { as } n \rightarrow \infty) \quad \Leftrightarrow \quad d\left(y_{i}^{n}, y_{i}\right) \rightarrow 0 \quad(\text { as } n \rightarrow \infty), \quad \forall i \in \Lambda_{k} .
$$


Definition 2.1 ([7]) Let $(R, \leq)$ be a partially ordered set and $d$ be a metric on $R$. We say that $(R, d, \leq)$ is regular if the following conditions hold:

(i) if $\left\{x_{n}\right\}$ is a non-decreasing sequence such that $x_{n} \rightarrow x$, then $x_{n} \leq x$ for all $n$,

(ii) if $\left\{y_{n}\right\}$ is a non-increasing sequence such that $y_{n} \rightarrow y$, then $y_{n} \geq y$ for all $n$.

Definition 2.2 ([2]) Let $(X, \leq)$ be a partially ordered set and $F: X^{2} \rightarrow X$. A mapping $F$ has the mixed monotone property if $F$ is monotone non-decreasing in its first argument and monotone non-increasing in its second argument, that is, for any $x, y \in X$,

$$
x_{1}, x_{2} \in X, \quad x_{1} \leq x_{2} \quad \text { implies } \quad F\left(x_{1}, y\right) \leq F\left(x_{2}, y\right),
$$

and

$$
y_{1}, y_{2} \in X, \quad y_{1} \leq y_{2} \quad \text { implies } \quad F\left(x, y_{1}\right) \geq F\left(x, y_{2}\right) .
$$

Definition 2.3 ([3]) Let $(X, \leq)$ be a partially ordered set and $F: X^{3} \rightarrow X$. A mapping $F$ has the mixed monotone property if $F(x, y, z)$ is monotone non-decreasing in $x$, monotone non-increasing in $y$, and monotone non-decreasing in $z$, that is, for any $x, y, z \in X$,

$$
\begin{array}{ll}
x_{1}, x_{2} \in X, & x_{1} \leq x_{2} \quad \Rightarrow \quad F\left(x_{1}, y, z\right) \leq F\left(x_{2}, y, z\right), \\
y_{1}, y_{2} \in X, & y_{1} \leq y_{2} \quad \Rightarrow \quad F\left(x, y_{1}, z\right) \geq F\left(x, y_{2}, z\right),
\end{array}
$$

and

$$
z_{1}, z_{2} \in X, \quad z_{1} \leq z_{2} \quad \Rightarrow \quad F\left(x, y, z_{1}\right) \leq F\left(x, y, z_{2}\right)
$$

Definition 2.4 ([22]) Let $(X, \leq)$ be a partially ordered set and $F: X^{n} \rightarrow X$. A mapping $F$ has the mixed monotone property if $F$ is monotone non-decreasing in arguments of $A$ and monotone non-increasing in arguments of $B$, i.e., for all $x_{1}, x_{2}, \ldots, x_{n}, y, z \in X$, and all $i$,

$$
y \leq z \quad \Rightarrow \quad F\left(x_{1}, \ldots, x_{i-1}, y, x_{i+1}, \ldots, x_{n}\right) \leq_{i} F\left(x_{1}, \ldots, x_{i-1}, z, x_{i+1}, \ldots, x_{n}\right) .
$$

Definition 2.5 Let $F: X^{k} \rightarrow X$ be a mapping. A point $\left(x_{1}, x_{2}, \cdots, x_{k}\right) \in X^{k}$ is:

(i) a coupled fixed point [2] if $k=2, F\left(x_{1}, x_{2}\right)=x_{1}$, and $F\left(x_{2}, x_{1}\right)=x_{2}$;

(ii) a tripled fixed point [3] if $k=3, F\left(x_{1}, x_{2}, x_{3}\right)=x_{1}, F\left(x_{2}, x_{1}, x_{2}\right)=x_{2}$, and $F\left(x_{3}, x_{2}, x_{1}\right)=x_{3}$

(iii) a $\Upsilon$-fixed point [25] of $F$ if $F\left(x_{\sigma_{i}(1)}, x_{\sigma_{i}(2)}, \ldots, x_{\sigma_{i}(k)}\right)=x_{i}$ for $i \in \Lambda_{k}$.

Definition 2.6 ([24]) Let $(X, \leq)$ be a partially ordered set. A mapping $f: X \rightarrow X$ is monotone non-decreasing if $x, y \in X, x \leq y \Rightarrow f(x) \leq f(y)$.

Definition 2.7 ([26]) An element $Y \in X^{k}$ is called a fixed point of the mapping $T: X^{k} \rightarrow$ $X^{k}$ if $T(Y)=Y$.

Lemma $2.8([27])$ Let $(X, \leq)$ be a partially ordered set and $d$ a metric on $X$. If $(X, \leq, d)$ is regular, then $\left(X^{k}, \preceq, \rho_{k}\right)$ is regular. 
Definition 2.9 A family of functions $f:[0, \infty)^{2} \rightarrow \mathbb{R}$ is called C-class if the following conditions hold:

(a) $f(s, t) \leq s$;

(b) $f$ is continuous;

(c) $f(s, t)=s$ implies that either $s=0$ or $t=0$.

For brevity, we denote the $C$-class by $\mathcal{C}$.

Example 2.10 ([28]) The following functions $f:[0, \infty)^{2} \rightarrow \mathbb{R}$ are elements of $\mathcal{C}$. For each $s, t \in[0, \infty)$ :

(1) $f(s, t)=s-t, f(s, t)=s \Rightarrow t=0$;

(2) $f(s, t)=\frac{s-t}{1+t}, f(s, t)=s \Rightarrow t=0$;

(3) $f(s, t)=\frac{s}{1+t}, f(s, t)=s \Rightarrow s=0$ or $t=0$;

(4) $f(s, t)=\log _{a} \frac{t+a^{s}}{1+t}, a>1, f(s, t)=s \Rightarrow s=0$ or $t=0$;

(5) $f(s, t)=k s, 0<k<1, f(s, t)=s \Rightarrow s=0$;

(6) $f(s, t)=(s+l) \frac{1}{1+t}-l, l>1, f(s, t)=s \Rightarrow t=0$;

(7) $f(s, t)=s \log _{a+t} a, a>1, f(s, t)=s \Rightarrow s=0$ or $t=0$;

(8) $f(s, t)=s-\frac{t}{1+t}, f(s, t)=s \Rightarrow t=0$;

(9) $f(s, t)=s-\left(\frac{1+s}{2+s}\right) t, f(s, t)=s \Rightarrow t=0$;

(10) $f(s, t)=\frac{s}{k+t}, k>1, f(s, t)=s \Rightarrow s=0$.

Remark 2.11 It is easy to verify that $C$-class is a natural generalization of classical Banach contraction principle (see (5) of Example 2.10).

Definition $2.12([29])$ An altering distance function is a function $\psi:[0, \infty) \rightarrow[0, \infty)$ which satisfies:

(a) $\psi$ is continuous and strictly increasing.

(b) $\psi(t)=0$ if and only if $t=0$.

Lemma 2.13 ([30]) Suppose that $(X, d)$ is a metric space. Let $\left\{x_{n}\right\}$ be a sequence in $X$ such that $d\left(x_{n}, x_{n+1}\right) \rightarrow 0$ as $n \rightarrow \infty$. If $\left\{x_{n}\right\}$ is not a Cauchy sequence then there exist an $\epsilon>0$ and sequences of positive integers $\{m(k)\}$ and $\{n(k)\}$ with $m(k)>n(k)>k$ such that $d\left(x_{m(k)}, x_{n(k)}\right) \geq \epsilon, d\left(x_{m(k)-1}, x_{n(k)}\right) \leq \epsilon$ and:

(i) $\lim _{k \rightarrow \infty} d\left(x_{m(k)-1}, x_{n(k)+1}\right)=\epsilon$;

(ii) $\lim _{k \rightarrow \infty} d\left(x_{m(k)}, x_{n(k)}\right)=\epsilon$;

(iii) $\lim _{k \rightarrow \infty} d\left(x_{m(k)-1}, x_{n(k)}\right)=\epsilon$.

Remark 2.14 Using a similar argument to the proof of Lemma 2.13, we get

$$
\lim _{k \rightarrow \infty} d\left(x_{n(k)+1}, x_{m(k)+1}\right)=\epsilon
$$

\section{Existence of fixed points}

In this section, we state and prove the existence of fixed points for non-decreasing and mixed monotone mappings with auxiliary functions in the setting of complete partially ordered metric spaces. In addition, the equivalence property between unidimensional and multidimensional fixed point theorems is investigated. 
We denote $\Phi$ the set of all continuous and strictly increasing functions $\varphi:[0, \infty) \rightarrow$ $[0, \infty)$, and $\Psi$ the set of all functions, such that $\lim _{t \rightarrow r} \psi(t)>0$ for every $r>0$ and $\psi(t)=$ $0 \Longleftrightarrow t=0$.

Theorem 3.1 Let $(X, \leq)$ be a partially ordered set and suppose that there is a metric $d$ on $X$ such that $(X, d)$ is a complete metric space. Assume that $T: X \rightarrow X$ is a non-decreasing mapping for which there exist $h \in \mathcal{C}, \varphi \in \Phi$, and $\psi \in \Psi$ such that, for all $y, v \in X$ with $y \geq v$,

$$
\varphi(d(T(y), T(v))) \leq h(\varphi(d(y, v)), \psi(d(y, v)))
$$

\section{Suppose that either}

(a) $T$ is continuous, or

(b) $(X, \leq, d)$ is regular.

If there exists $z_{0} \in X$ such that $z_{0} \asymp T\left(z_{0}\right)$, then $T$ has a fixed point.

Proof Starting with $z_{0}$ in $X$, define the sequence $\left\{z_{n}\right\} \subset X$ by $z_{n+1}=T\left(z_{n}\right)$ for $n \geq 0$. Obviously, if $z_{n_{0}+1}=z_{n_{0}}$ for some $n_{0} \geq 0$, then $z_{n_{0}}$ is a fixed point of $T$. So, assume that $z_{n+1} \neq z_{n}$ for every $n \geq 0$.

Since $z_{0} \asymp T\left(z_{0}\right)$, without loss of generality, we may assume that $z_{0} \leq T\left(z_{0}\right)$ (the case $z_{0} \geq T\left(z_{0}\right)$ is treated similarly), that is, $z_{0} \leq z_{1}$. Since $T$ is a non-decreasing mapping, it is easy to verify that the sequence $\left\{z_{n}\right\}_{n=0}^{\infty}$ is non-decreasing. Taking $y=z_{n}$ and $v=z_{n-1}$ in (3), we obtain

$$
\begin{aligned}
\varphi\left(d\left(z_{n+1}, z_{n}\right)\right) & =\varphi\left(d\left(T\left(z_{n}\right), T\left(z_{n-1}\right)\right)\right) \\
& \leq h\left(\varphi\left(d\left(z_{n}, z_{n-1}\right)\right), \psi\left(d\left(z_{n}, z_{n-1}\right)\right)\right) \\
& \leq \varphi\left(d\left(z_{n}, z_{n-1}\right)\right)
\end{aligned}
$$

for all $n \geq 1$.

Since $\varphi$ is strictly increasing, we have

$$
d\left(z_{n+1}, z_{n}\right) \leq d\left(z_{n}, z_{n-1}\right), \quad \forall n \geq 1 .
$$

Hence, the sequence $\left\{\delta_{n}\right\}_{n=0}^{\infty}$ given by $\delta_{n}=d\left(z_{n+1}, z_{n}\right)$ is a monotone decreasing and bounded below. Therefore, there exists some $\delta \geq 0$ such that $\lim _{n \rightarrow \infty} \delta_{n}=\delta$. Now, we shall prove that $\delta=0$. Assume that $\delta>0$. Using Definition 2.9, we know that when $h(s, t)=s$, then $s=0$ or $t=0$ and $h(s, t)<s$ when $s>0$ and $t>0$. Using the properties of $\varphi$ and $\psi$, we have $\varphi(\delta)>\varphi(0) \geq 0$ and $\lim _{n \rightarrow \infty} \psi\left(\delta_{n-1}\right)>0$. Therefore, by letting $n \rightarrow \infty$ in (4) and using the properties of $h$, we have

$$
\varphi(\delta) \leq h\left(\varphi(\delta), \lim _{n \rightarrow \infty} \psi\left(\delta_{n-1}\right)\right)<\varphi(\delta),
$$

which is a contradiction. Thus, $\lim _{n \rightarrow \infty} \delta_{n}=0$.

Suppose that $\left\{z_{n}\right\}$ is not a Cauchy sequence. Using Lemma 2.13, we see that there exist $\epsilon>0$ and two sequences $\{n(t)\}$ and $\{m(t)\}$ of positive integers such that, for all $t \in \mathbb{N}$, $m(t)>n(t) \geq t$ and

$$
\lim _{t \rightarrow \infty} d\left(z_{m(t)}, z_{n(t)}\right)=\lim _{t \rightarrow \infty} d\left(z_{m(t)+1}, z_{n(t)+1}\right)=\epsilon .
$$


Since $n(t)<m(t)$, we have $z_{m(t)} \geq z_{n(t)}$. Hence, using (3) with $v=z_{n(t)}$ and $y=z_{m(t)}$, we obtain

$$
\varphi\left(d\left(z_{n(t)+1}, z_{m(t)+1}\right)\right) \leq h\left(\varphi\left(d\left(z_{n(t)}, z_{m(t)}\right)\right), \psi\left(d\left(z_{n(t)}, z_{m(t)}\right)\right)\right)
$$

Using the properties of $\varphi$ and $\psi$, we have $\varphi(\epsilon)>0$ and $\lim _{t \rightarrow \infty} \psi\left(r_{t}\right)>0$, where $r_{t}=$ $d\left(z_{n(t)}, z_{m(t)}\right)$. Letting $t \rightarrow \infty$ in (6) and using (5), we have

$$
\varphi(\epsilon) \leq h\left(\varphi(\epsilon), \lim _{t \rightarrow \infty} \psi\left(r_{t}\right)\right)<\varphi(\epsilon)
$$

which is a contradiction. Hence, the sequence $\left\{z_{n}\right\}_{n=0}^{\infty}$ is a Cauchy sequence in the metric space $(X, d)$. Since $(X, d)$ is complete, there exists $\bar{z} \in X$ such that $\lim _{n \rightarrow \infty} z_{n}=\bar{z}$.

Now suppose that (a) holds. It follows from $z_{n+1}=T\left(z_{n}\right)$ that $\bar{z}$ is a fixed point of $T$, that is, $T(\bar{z})=\bar{z}$.

Suppose that (b) holds. Since $\left\{z_{n}\right\}_{n=0}^{\infty}$ is a non-decreasing sequence that converges to $\bar{z}$, we have $z_{n} \leq \bar{z}$ for all $n \geq 0$. From (3) and the fact that $h \in \mathcal{C}$, we obtain

$$
\varphi\left(d\left(z_{n+1}, T(\bar{z})\right)\right)=\varphi\left(d\left(T\left(z_{n}\right), T(\bar{z})\right)\right) \leq h\left(\varphi\left(d\left(z_{n}, \bar{z}\right)\right), \psi\left(d\left(z_{n}, \bar{z}\right)\right)\right) \leq \varphi\left(d\left(z_{n}, \bar{z}\right)\right)
$$

for all $n \geq 0$. From (7) and the strict monotonicity of $\varphi$, we have

$$
d\left(z_{n+1}, T(\bar{z})\right) \leq d\left(z_{n}, \bar{z}\right)
$$

Letting $n \rightarrow \infty$ in (8) and using $\lim _{n \rightarrow \infty} z_{n}=\bar{z}$, we get $d(\bar{z}, T(\bar{z})) \leq d(\bar{z}, \bar{z})=0$, and so $d(\bar{z}, T(\bar{z}))=0$, which implies $\bar{z}=T(\bar{z})$.

Remark 3.2 In Theorem 3.1, we use $h \in \mathcal{C}$, and $\mathcal{C}$ is a class of more general functions than the gauge function used in Theorems 2.1 and 2.2 of [24]. Indeed, the gauge function, $h(s, t)=s-t$ in Theorems 2.1 and 2.2 of [24] is an element of $\mathcal{C}$.

Taking $h(s, t)=s-t$ or $h(s, t)=\frac{s}{1+t}$ in Theorem 3.1, we obtain the following results immediately.

Corollary 3.3 Let $(X, \leq)$ be a partially ordered set and suppose that there is a metric $d$ on $X$ such that $(X, d)$ is a complete metric space. Assume that $T: X \rightarrow X$ is a non-decreasing mapping for which there exist $\varphi \in \Phi$ and $\psi \in \Psi$ such that, for all $y, v \in X$ with $y \geq v$,

$$
\varphi(d(T(y), T(v))) \leq \varphi(d(y, v))-\psi(d(y, v)) .
$$

\section{Suppose that either}

(a) $T$ is continuous, or

(b) $(X, \leq, d)$ is regular.

If there exists $z_{0} \in X$ such that $z_{0} \asymp T\left(z_{0}\right)$, then $T$ has a fixed point.

Remark 3.4 We note that, if $\psi$ is an altering distance function, then $\psi \in \Psi$. But the reverse is not true in general (see Example 3.5). Therefore, Corollary 3.3 generalizes the well-known results of Harjani and Sadarangani's [24] in the framework of partially ordered metric spaces (see Theorems 2.1 and 2.2 in [24]). 
Example 3.5 Let $X=\{0,0.5,1,1.5,2\}$ with the usual metric $d(x, y)=|x-y|$, for all $x, y \in X$. We consider the following order relation on $X$ :

$$
x, y \in X, \quad x \leq y \quad \Leftrightarrow \quad x=y \text { or }(x, y)=(0,2) .
$$

Consider $T: X \rightarrow X$ and $\psi, \varphi:[0, \infty) \rightarrow[0, \infty)$ defined by

$$
T(x)=\left\{\begin{array}{ll}
0, & \text { if } x \in\{0,0.5,2\}, \\
1, & \text { otherwise; }
\end{array} \quad \psi(t)=\left\{\begin{array}{ll}
\frac{1}{t}, & \text { if } t>0, \\
0, & \text { if } t=0 ;
\end{array} \quad \varphi(t)=t .\right.\right.
$$

It is easy to verify the following statements.

(1) $(X, d)$ is a complete metric space and $(X, \leq, d)$ is regular.

(2) $T$ is a continuous mapping.

(3) $\varphi \in \Phi$ and $\psi \in \Psi$.

(4) Take $z_{0}=1$. Then $1=T(1)$.

(5) $T$ is a non-decreasing mapping. Indeed, let $x, y \in X$ such that $x \leq y$, (a) if $x=y$, then $T(x)=T(y)$; (b) if $(x, y)=(0,2)$, then $T(x)=T(y)$. Therefore, $T$ is a non-decreasing mapping.

(6) $T$ satisfies (9). In fact, it is evident that (9) holds when $y=v$. If $(v, y)=(0,2)$, then

$$
\varphi(d(T(y), T(v)))=\varphi(d(0,0))=0 \leq 1.5=\varphi(2)-\psi(2)=\varphi(d(y, v))-\psi(d(y, v)) .
$$

This shows that all the conditions of Corollary 3.3 are satisfied. Therefore by using Corollary 3.3, $T$ has a fixed point. Indeed, 0 and 1 are two fixed points of $T$. However, Theorems 2.1 and 2.2 of Harjani and Sadarangani's [24] cannot be applied to this example because $\psi$ is not an altering distance function.

Corollary 3.6 Let $(X, \leq)$ be a partially ordered set and suppose that there is a metric $d$ on $X$ such that $(X, d)$ is a complete metric space. Assume that $T: X \rightarrow X$ is a non-decreasing mapping for which there exist $\varphi \in \Phi$ and $\psi \in \Psi$ such that, for all $y, v \in X$ with $y \geq v$,

$$
\varphi(d(T(y), T(v))) \leq \frac{\varphi(d(y, v))}{1+\psi(d(y, v))} .
$$

\section{Suppose that either}

(a) $T$ is continuous, or

(b) $(X, \leq, d)$ is regular.

If there exists $z_{0} \in X$ such that $z_{0} \asymp T\left(z_{0}\right)$, then $T$ has a fixed point.

Example 3.7 Let $(X, \leq)$ be the partially ordered set with $X=[0, \infty)$ and the natural ordering of the real number as the partial ordering $\leq$. Consider the metric on $X: d(x, y)=|x-y|$ for all $x, y \in X$. Then $(X, d)$ is complete and $(X, \leq, d)$ is regular.

Let $T: X \rightarrow X$ be defined by $T(x)=\frac{x}{1+x}$ for all $x \in X$. Then $T$ is a non-decreasing and continuous mapping. Consider $\psi, \varphi:[0, \infty) \rightarrow[0, \infty)$ defined by $\psi(t)=\varphi(t)=t$. It is obvious that $\varphi \in \Phi$ and $\psi \in \Psi$.

We now show that $T$ satisfies (10). Consider

$$
|T(x)-T(y)|=\frac{|x-y|}{1+x+y+x y}=\frac{|x-y|}{1+|x-y|+2 \min \{x, y\}+x y} \leq \frac{|x-y|}{1+|x-y|} .
$$


This shows that all the conditions of Corollary 3.6 are satisfied and hence $T$ has a fixed point. Indeed, 0 is a unique fixed points of $T$. However, Theorems 2.1 and 2.2 of Harjani and Sadarangani's [24] cannot be applied to this example because the $T$ does not satisfy the inequality $\varphi(d(T(x), T(y))) \leq \varphi(d(x, y))-\psi(d(x, y))$ for all $x \geq y$. Indeed, if $x \neq y$, then

$$
\varphi(d(T(x), T(y)))=\frac{|x-y|}{1+x+y+x y}>0=\varphi(d(x, y))-\psi(d(x, y)) .
$$

Now we give the following multidimensional fixed point theorem for mixed monotone mappings.

Theorem 3.8 Let $(X, \leq)$ be a partially ordered set and suppose that there is a metric $d$ on $X$ such that $(X, d)$ is a complete metric space. Suppose that $\Upsilon=\left(\sigma_{1}, \sigma_{2}, \ldots, \sigma_{k}\right)$ is a $k$ tuple of mapping from $\Lambda_{k}$ into itself such that $\sigma_{i} \in \Omega_{A, B}$ if $i \in A$ and $\sigma_{i} \in \Omega_{A, B}^{\prime}$ if $i \in B$ and $F: X^{k} \rightarrow X$ is a mixed monotone mapping and there exist $h \in \mathcal{C}, \varphi \in \Phi$, and $\psi \in \Psi$ such that

$$
\begin{aligned}
\varphi( & \left.\frac{1}{k} \sum_{i=1}^{k} d\left(F\left(x_{\sigma_{i}(1)}, x_{\sigma_{i}(2)}, \ldots, x_{\sigma_{i}(k)}\right), F\left(y_{\sigma_{i}(1)}, y_{\sigma_{i}(2)}, \ldots, y_{\sigma_{i}(k)}\right)\right)\right) \\
& \leq h\left(\varphi\left(\frac{1}{k} \sum_{i=1}^{k} d\left(x_{i}, y_{i}\right)\right), \psi\left(\frac{1}{k} \sum_{i=1}^{k} d\left(x_{i}, y_{i}\right)\right)\right)
\end{aligned}
$$

for which $x_{i} \leq_{i} y_{i}$ for $i \in \Lambda_{k}$. Further assume that either $F$ is continuous or $(X, \leq, d)$ is regular. If there exist $x_{0}^{1}, x_{0}^{2}, \ldots, x_{0}^{k} \in X$ such that either of the following conditions is satisfied:

$$
x_{0}^{i} \leq_{i} F\left(x_{0}^{\sigma_{i}(1)}, x_{0}^{\sigma_{i}(2)}, \ldots, x_{0}^{\sigma_{i}(k)}\right) \quad \text { for } i \in \Lambda_{k} \text {, }
$$

or

$$
x_{0}^{i} \geq_{i} F\left(x_{0}^{\sigma_{i}(1)}, x_{0}^{\sigma_{i}(2)}, \ldots, x_{0}^{\sigma_{i}(k)}\right) \quad \text { for } i \in \Lambda_{k} .
$$

Then F has one $\Upsilon$-fixed point.

Proof Let $T: X^{k} \rightarrow X^{k}$ be defined by

$$
\begin{aligned}
T(Y)= & \left(F\left(y_{\sigma_{1}(1)}, y_{\sigma_{1}(2)}, \ldots, y_{\sigma_{1}(k)}\right), \ldots, F\left(y_{\sigma_{i}(1)}, y_{\sigma_{i}(2)}, \ldots, y_{\sigma_{i}(k)}\right), \ldots\right. \\
& \left.F\left(y_{\sigma_{k}(1)}, y_{\sigma_{k}(2)}, \ldots, y_{\sigma_{k}(k)}\right)\right)
\end{aligned}
$$

for $Y \in X^{k}$. Using similar arguments to the proof of Corollary 3.11 in [26], $T$ is a nondecreasing mapping.

Using the inequalities (1), (11) and (14), we have

$$
\varphi\left(\rho_{k}(T(Y), T(V))\right) \leq h\left(\varphi\left(\rho_{k}(Y, V)\right), \psi\left(\rho_{k}(Y, V)\right)\right),
$$

where $\rho_{k}$ is defined by (2). Since $(X, \leq, d)$ is a complete partially ordered metric space, so is $\left(X^{k}, \preceq, \rho_{k}\right)$. As $F$ is continuous and using inequality (14), $T$ is continuous. Using 
Lemma 2.8, we have $\left(X^{k}, \preceq, \rho_{k}\right)$ is regular. From (12) and (13), there exists $X_{0} \in X^{k}$ such that $X_{0} \asymp T\left(X_{0}\right)$. Therefore, by using Theorem 3.1, $T$ has a fixed point. Hence $F$ has a $\Upsilon$-fixed point.

Remark 3.9 Note that the multidimensional fixed point theorem (Theorem 3.8) is equivalent to the unidimensional fixed point theorem (Theorem 3.1). Also, we find that Theorem 3.8 is a consequence of Theorem 3.1. Conversely, taking $k=1, A=\{1\}, B=\emptyset$, and $F=T$ in Theorem 3.8, we obtain Theorem 3.1 immediately.

Using similar arguments to the proof of Theorem 3.8, the following results are immediate consequences of the unidimensional fixed point theorem (Theorem 3.1).

Corollary 3.10 Let $(X, \leq)$ be a partially ordered set and suppose that there is a metric $d$ on $X$ such that $(X, d)$ is a complete metric space. Assume that $F: X^{2} \rightarrow X$ is a mixed monotone mapping for which there exist $h \in \mathcal{C}, \varphi \in \Phi$, and $\psi \in \Psi$ such that, for all $x, y, u, v \in X$ with $x \geq u, y \leq v$,

$$
\begin{aligned}
\varphi\left(\frac{d(F(x, y), F(u, v))+d(F(y, x), F(v, u))}{2}\right) \leq & h\left(\varphi\left(\frac{d(x, u)+d(y, v)}{2}\right),\right. \\
& \left.\psi\left(\frac{d(x, u)+d(y, v)}{2}\right)\right) .
\end{aligned}
$$

Suppose that either

(a) $F$ is continuous, or

(b) $(X, d, \leq)$ is regular.

If there exist $x_{0}, y_{0} \in X$ such that

$$
x_{0} \leq F\left(x_{0}, y_{0}\right) \text { and } y_{0} \geq F\left(y_{0}, x_{0}\right)
$$

or

$$
x_{0} \geq F\left(x_{0}, y_{0}\right) \quad \text { and } \quad y_{0} \leq F\left(y_{0}, x_{0}\right)
$$

then there exist $\bar{x}, \bar{y} \in X$ such that $\bar{x}=F(\bar{x}, \bar{y})$ and $\bar{y}=F(\bar{y}, \bar{x})$, that is, $F$ has a coupled fixed point.

Corollary 3.11 Let $(X, \leq)$ be a partially ordered set and suppose that there is a metric $d$ on $X$ such that $(X, d)$ is a complete metric space. Assume that $F: X^{3} \rightarrow X$ is a mixed monotone mapping for which there exist $h \in \mathcal{C}, \varphi \in \Phi$, and $\psi \in \Psi$ such that, for all $x, y, z, u, v, w \in X$ with $x \geq u, y \leq v, z \geq w$,

$$
\begin{gathered}
\varphi\left(\frac{d(F(x, y, z), F(u, v, w))+d(F(y, x, y), F(v, u, v))+d(F(z, y, x), F(w, v, u))}{3}\right) \\
\leq h\left(\varphi\left(\frac{d(x, u)+d(y, v)+d(z, w)}{3}\right), \psi\left(\frac{d(x, u)+d(y, v)+d(z, w)}{3}\right)\right) .
\end{gathered}
$$

Suppose that either 
(a) $F$ is continuous, or

(b) $(X, \leq, d)$ is regular.

If there exist $x_{0}, y_{0}, z_{0} \in X$ such that

$$
x_{0} \leq F\left(x_{0}, y_{0}, z_{0}\right), \quad y_{0} \geq F\left(y_{0}, x_{0}, y_{0}\right) \quad \text { and } \quad z_{0} \leq F\left(z_{0}, y_{0}, x_{0}\right) \text {, }
$$

or

$$
x_{0} \geq F\left(x_{0}, y_{0}, z_{0}\right), \quad y_{0} \leq F\left(y_{0}, x_{0}, y_{0}\right) \quad \text { and } \quad z_{0} \geq F\left(z_{0}, y_{0}, x_{0}\right) \text {, }
$$

then there exist $x, y, z \in X$ such that

$$
F(x, y, z)=x, \quad F(y, x, y)=y \quad \text { and } \quad F(z, y, x)=z \text {, }
$$

that is, F has a tripled fixed point.

Using Theorem 3.1, we obtain the following result on multidimensional fixed points, which generalizes Theorem 3.1 of Wang [26].

Theorem 3.12 Let $(X, \leq)$ be a partially ordered set and suppose that there is a metric $d$ on $X$ such that $(X, d)$ is a complete metric space. Assume that $T: X^{k} \rightarrow X^{k}$ is a non-decreasing mapping for which there exist $h \in \mathcal{C}, \varphi \in \Phi$, and $\psi \in \Psi$ such that, for all $Y, V \in X^{k}$ with $Y \succeq V$,

$$
\varphi\left(\rho_{k}(T(Y), T(V))\right) \leq h\left(\varphi\left(\rho_{k}(Y, V)\right), \psi\left(\rho_{k}(Y, V)\right)\right)
$$

where $\rho_{k}$ is defined by (2). Suppose that either

(a) $T$ is continuous, or

(b) $(X, \leq, d)$ is regular.

If there exists $Z_{0} \in X^{k}$ such that $Z_{0} \asymp T\left(Z_{0}\right)$, then $T$ has a fixed point.

Proof Using Lemma 2.8, we find that $\left(X^{k}, \preceq, \rho_{k}\right)$ is regular. By our assumptions, all the conditions of Theorem 3.1 are satisfied in the setting of a complete partially ordered metric space $\left(X^{k}, \preceq, \rho_{k}\right)$. Therefore, by using Theorem $3.1, T$ has a fixed point.

Remark 3.13 (a) The metric $\rho_{k}$ in Theorem 3.12 can be replaced by some other metrics on $X^{k}$, for example,

$$
\bar{\rho}_{k}(Y, V)=\max _{1 \leq i \leq k} d\left(y_{i}, v_{i}\right)
$$

where $Y, V \in X^{k}$.

(b) We also find that the multidimensional fixed point theorem (Theorem 3.12) is equivalent to the unidimensional fixed point theorem (Theorem 3.1). In fact, Theorem 3.12 is a consequence of Theorem 3.1. Conversely, taking $k=1$ in Theorem 3.12, we obtain Theorem 3.1 immediately. 


\section{Uniqueness of fixed points}

Now, we state and prove the uniqueness of fixed points in the setting of a complete partially ordered metric space.

Theorem 4.1 In addition to the hypotheses of Theorem 3.1, suppose that, for all fixed points $\bar{y}, y^{*} \in X$ of $T$, there exists $z \in X$ such that $z$ is comparable to $\bar{y}$ and to $y^{*}$. Then $T$ has a unique fixed point.

Proof From Theorem 3.1, the set of fixed points of $T$ is nonempty. Assume that $\bar{y}$ and $y^{*}$ are two fixed points of $T$. Put $z_{0}=z$ and $z_{n+1}=T\left(z_{n}\right)$ for $n \geq 0$. Since $z$ is comparable to $\bar{y}$, we may assume $z \leq \bar{y}$. Since $T$ is a non-decreasing mapping, we obtain inductively $z_{n} \leq \bar{y}$ for $n \geq 0$. Therefore, by using (3), we have

$$
\varphi\left(d\left(\bar{y}, z_{n+1}\right)\right)=\varphi\left(d\left(T(\bar{y}), T\left(z_{n}\right)\right)\right) \leq h\left(\varphi\left(d\left(\bar{y}, z_{n}\right)\right), \psi\left(d\left(\bar{y}, z_{n}\right)\right)\right)
$$

which implies that

$$
\varphi\left(d\left(\bar{y}, z_{n+1}\right)\right) \leq \varphi\left(d\left(\bar{y}, z_{n}\right)\right) .
$$

Using the inequality (16) and the strict monotonicity of $\varphi$, the sequence $\left\{\Delta_{n}\right\}$ defined by $\Delta_{n}=d\left(\bar{y}, z_{n}\right)$ is non-increasing. Hence, there exists $\beta \geq 0$ such that $\lim _{n \rightarrow \infty} \Delta_{n}=\beta$. Now we shall prove that $\beta=0$. Suppose, conversely, that $\beta>0$. Using the properties of $\varphi$ and $\psi$, we have $\varphi(\beta)>0$ and $\lim _{\Delta_{n} \rightarrow \beta} \psi\left(\Delta_{n}\right)>0$. Letting $n \rightarrow \infty$ in (15), we get

$$
\varphi(\beta) \leq h\left(\varphi(\beta), \lim _{\Delta_{n} \rightarrow \beta} \psi\left(\Delta_{n}\right)\right)<\varphi(\beta),
$$

which is a contradiction. Thus $\beta=0$, that is,

$$
\lim _{n \rightarrow \infty} d\left(\bar{y}, z_{n}\right)=0
$$

Similarly, we obtain

$$
\lim _{n \rightarrow \infty} d\left(y^{*}, z_{n}\right)=0
$$

Combining (17) and (18) yields $y^{*}=\bar{y}$.

Using similar arguments to the proof of Theorem 3.8, we deduce the following corollaries from Theorem 4.1.

Corollary 4.2 In addition to the hypotheses of Theorem 3.8, suppose that, for all $\Upsilon$-fixed points $\bar{Y}, Y^{*} \in X^{k}$ of $F$, there exists $Z \in X^{k}$ such that $Z$ is comparable to $\bar{Y}$ and to $Y^{*}$. Then $F$ has a unique $\Upsilon$-fixed point.

Corollary 4.3 In addition to the hypotheses of Corollary 3.10, suppose that, for all coupled fixed points $\bar{Y}, Y^{*} \in X^{2}$ of $F$, there exists $Z \in X^{2}$ such that $Z$ is comparable to $\bar{Y}$ and to $Y^{*}$. Then $F$ has a unique coupled fixed point. 
Corollary 4.4 In addition to the hypotheses of Corollary 3.11, suppose that, for all tripled fixed points $\bar{Y}, Y^{*} \in X^{3}$ of $F$, there exists $Z \in X^{3}$ such that $Z$ is comparable to $\bar{Y}$ and to $Y^{*}$. Then $F$ has a unique tripled fixed point.

\section{Application to integral equations}

In this section, we present two examples where our main results can be applied. Consider the integral equation

$$
x(t)=\int_{0}^{T} K(t, s, x(s)) d s+g(t), \quad t \in[0, T],
$$

where $T>0$. Consider $C[0, T]=\{x:[0, T] \rightarrow \mathbb{R}: x$ is continuous on $[0, T]\}$ equipped with the metric

$$
d(x, y)=\sup _{t \in[0, T]}|x(t)-y(t)|, \quad \text { for each } x, y \in C[0, T] .
$$

It is clear that $(C[0, T], d)$ is a complete metric space. $C[0, T]$ can be equipped with the following partial order:

$$
x, y \in C[0, T], \quad x \leq y \quad \Leftrightarrow \quad x(t) \leq y(t), \quad \text { for each } t \in[0, T] .
$$

Clearly, $(C[0, T], \leq)$ satisfies the following condition (see [24]):

$$
\text { for } x, y \in X \text { there exists a lower bound or an upper bound. }
$$

Due to [31], we know that $(C[0, T], d, \leq)$ is regular and condition (20) is equivalent to

$$
\text { for } x, y \in X \text { there exists } z \in X \text { which is comparable to } x \text { and } y \text {. }
$$

Theorem 5.1 Assume that the following hypotheses hold:

(i) $K:[0, T] \times[0, T] \times \mathbb{R} \rightarrow[0, \infty)$ and $g:[0, T] \rightarrow[0, \infty)$ are continuous,

(ii) there exist $h \in \mathcal{C}, \psi \in \Psi$, and a continuous function $\tilde{G}:[0, T] \times[0, T] \rightarrow[0, \infty)$ such that

$$
0 \leq K(t, s, y)-K(t, s, x) \leq \tilde{G}(t, s) h(d(x, y), \psi(d(x, y)))
$$

for all $s, t \in[0, T]$ and $x, y \in C[0, T]$ with $x \leq y$;

(iii) $\sup _{t \in[0, T]} \int_{0}^{T} \tilde{G}(t, s) d s \leq 1$ or $\sup _{t \in[0, T]}\left(\int_{0}^{T} \tilde{G}(t, s)^{2} d s\right)^{\frac{1}{2}} \leq \frac{1}{\sqrt{T}}$.

Then the integral equation (19) has a unique non-negative solution $u^{*} \in C[0, T]$.

Proof Let $H: C[0, T] \rightarrow C[0, T]$ be the following function:

$$
(H x)(t)=\int_{0}^{T} K(t, s, x(s)) d s+g(t), \quad t \in[0, T], x \in C[0, T] .
$$

Assume that $x \leq y$. From (ii), for all $s, t \in[0, T]$, we have $K(t, s, x(s)) \leq K(t, s, y(s))$. So

$$
(H x)(t)=\int_{0}^{T} K(t, s, x(s)) d s+g(t) \leq \int_{0}^{T} K(t, s, y(s)) d s+g(t)=(H y)(t),
$$


that is, $H$ is a non-decreasing mapping. Suppose that $\sup _{t \in[0, T]} \int_{0}^{T} \tilde{G}(t, s) d s \leq 1$. It follows from (ii) that

$$
\begin{aligned}
d(H x, H y) & =\sup _{t \in[0, T]}|(H x)(t)-(H y)(t)| \\
& =\sup _{t \in[0, T]} \int_{0}^{T}(K(t, s, y(s))-K(t, s, x(s))) d s \\
& \leq \sup _{t \in[0, T]} \int_{0}^{T} \tilde{G}(t, s) h(d(x, y), \psi(d(x, y))) d s \\
& \leq h(d(x, y), \psi(d(x, y))) \sup _{t \in[0, T]} \int_{0}^{T} \tilde{G}(t, s) d s \\
& \leq h(d(x, y), \psi(d(x, y)))
\end{aligned}
$$

for all $x \leq y$.

Now suppose that $\sup _{t \in[0, T]}\left(\int_{0}^{T} \tilde{G}(t, s)^{2} d s\right)^{\frac{1}{2}} \leq \frac{1}{\sqrt{T}}$. From (ii) and Cauchy-Schwarz inequality, we see that

$$
\begin{aligned}
d(H x, H y) & \leq \sup _{t \in[0, T]} \int_{0}^{T} \tilde{G}(t, s) h(d(x, y), \psi(d(x, y))) d s \\
& \leq \sup _{t \in[0, T]}\left(\int_{0}^{T} \tilde{G}(t, s)^{2} d s\right)^{\frac{1}{2}}\left(\int_{0}^{T}(h(d(x, y), \psi(d(x, y))))^{2} d s\right)^{\frac{1}{2}} \\
& \leq \sup _{t \in[0, T]}\left(\int_{0}^{T} \tilde{G}(t, s)^{2} d s\right)^{\frac{1}{2}} \sqrt{T} h(d(x, y), \psi(d(x, y))) \\
& \leq h(d(x, y), \psi(d(x, y)))
\end{aligned}
$$

for all $x \leq y$. Now, if choose $\varphi(t)=t$, then we get

$$
\varphi(d(H(x), H(y))) \leq h(\varphi(d(x, y)), \psi(d(x, y)))
$$

for all $x, y \in C[0, T]$ with $x \leq y$. Finally, as $K$ and $g$ are non-negative functions, we obtain

$$
H 0=\int_{0}^{T} K(t, s, 0) d s+g(t) \geq 0
$$

Therefore by using Theorems 3.1 and $4.1, H$ has a unique fixed point $u^{*} \in C[0, T]$ and $u^{*}$ is a unique non-negative solution of (19).

Consider the following two-point boundary value problem of second order differential equation:

$$
\left\{\begin{array}{l}
-\frac{d^{2} x}{d t^{2}}=f(t, x), \quad x \in[0, \infty), t \in[0,1] \\
x(0)=x(1)=0 .
\end{array}\right.
$$


It is well known that $x \in C^{2}[0,1]$ being a solution of (21) is equivalent to $x \in C^{2}[0,1]$ being a solution of the integral equation

$$
x(t)=\int_{0}^{1} G(t, s) f(s, x(s)) d s, \quad \text { for } t \in[0,1]
$$

where $G(t, s)$ is the Green function given by

$$
G(t, s)= \begin{cases}t(1-s), & 0 \leq t \leq s \leq 1 \\ s(1-t), & 0 \leq s \leq t \leq 1\end{cases}
$$

Corollary 5.2 ([24]) Consider problem (21) with $f:[0,1] \times \mathbb{R} \rightarrow[0, \infty)$ continuous and non-decreasing with respect to the second variable and suppose that there exists $0<\alpha \leq 8$ such that for $x, y \in \mathbb{R}$ with $y \geq x$

$$
f(t, y)-f(t, x) \leq \alpha \sqrt{\ln \left[(y-x)^{2}+1\right]} .
$$

Then our problem (21) has a unique non-negative solution.

Proof Take $K(t, s, x(s))=G(t, s) f(s, x(s))$ and $g(t)=0$ for all $s, t \in[0,1]$. It is clear that condition (i) of Theorem 5.1 holds since $f(t, x)$ and $G(t, s)$ are non-negative continuous functions.

Now we show that condition (ii) holds. As $f$ is non-decreasing with respect to the second variable, for all $s, t \in[0,1]$ and $x, y \in C[0,1]$ with $x \leq y$, we have

$$
K(t, s, x(s)) \leq K(t, s, y(s))
$$

It follows from (22) that

$$
\begin{aligned}
K(t, s, y)-K(t, s, x) & \leq G(t, s)(f(s, y(s))-f(s, x(s))) \\
& \leq \alpha G(t, s) \sqrt{\ln \left[(y-x)^{2}+1\right]} \\
& \leq \alpha G(t, s) \sqrt{\ln \left[d(y, x)^{2}+1\right]}
\end{aligned}
$$

for all $s, t \in[0,1]$ and $x, y \in C[0,1]$ with $x \leq y$. If we choose $h(s, t)=\sqrt{\ln \left(s^{2}+1\right)}$ and $\tilde{G}(t, s)=$ $\alpha G(t, s)$, then condition (ii) of Theorem 5.1 holds.

It is easy to verify that $\int_{0}^{1} G(t, s) d s=-\frac{t^{2}}{2}+\frac{t}{2}$ and that

$$
\sup _{t \in[0,1]} \int_{0}^{1} \tilde{G}(t, s) d s=\alpha \sup _{t \in[0,1]} \int_{0}^{1} G(t, s) d s \leq 1 .
$$

Therefore, all hypotheses of Theorem 5.1 are satisfied. Thus our problem (21) has a unique non-negative solution. 
Authors' contributions

The authors read and approved the final manuscript.

\section{Author details}

${ }^{1}$ School of Mathematical Sciences, Yancheng Teachers University, Yancheng, Jiangsu 224051, P.R. China. ${ }^{2}$ Department of Mathematics, Karaj Branch, Islamic Azad University, Karaj, Iran. ${ }^{3}$ School of Mathematics, Thapar University, Patiala, Punjab 147004 , India.

\section{Acknowledgements}

The authors are grateful to the anonymous referees for their helpful comments which improved the presentation of the original version of this paper. This work was supported by the Natural Science Foundation of Jiangsu Province under grant (13KJB110028)

\section{Received: 20 March 2015 Accepted: 4 November 2015 Published online: 17 November 2015}

\section{References}

1. Guo, D, Lakshmikantham, V: Coupled fixed points of nonlinear operators with applications. Nonlinear Anal. 11, 623-632 (1987)

2. Gnana Bhaskar, T, Lakshmikantham, V: Fixed point theorems in partially ordered metric spaces and applications. Nonlinear Anal. 65(7), 1379-1393 (2006)

3. Berinde, V, Borcut, M: Tripled fixed point theorems for contractive type mappings in partially ordered metric spaces. Nonlinear Anal. 74, 4889-4897 (2011)

4. Berinde, $\mathrm{V}$ : Coupled fixed point theorems for $\phi$-contractive mixed monotone mappings in partially ordered metric spaces. Nonlinear Anal. 75(6), 3218-3228 (2012)

5. Berinde, V: Generalized coupled fixed point theorems for mixed monotone mappings in partially ordered metric spaces. Nonlinear Anal. 74, 7347-7355 (2011)

6. Luong, NV, Thuan, NX: Coupled fixed points in partially ordered metric spaces and application. Nonlinear Anal. 74 983-992 (2011)

7. Aydi, H, Karapınar, E, Postolache, M: Tripled coincidence theorems for weak $\varphi$-contractions in partially ordered metric spaces. Fixed Point Theory Appl. (2012). doi:10.1186/1687-1812-2012-44

8. Lakshmikantham, V, Ćirić, L: Coupled fixed point theorems for nonlinear contractions in partially ordered metric spaces. Nonlinear Anal. 70, 4341-4349 (2009)

9. Berinde, V: Coupled coincidence point theorems for mixed point monotone nonlinear operators. Comput. Math. Appl. 64(6), 1770-1777 (2012)

10. Agarwal, RP, El-Gebeily, MA, O’Regan, D: Generalized contractions in partially ordered metric spaces. Appl. Anal. 87, 1-8 (2008)

11. Borcut, $M$, Berinde, $\mathrm{V}$ : Tripled coincidence theorems for contractive type mappings in partially ordered metric spaces. Appl. Math. Comput. 218(10), 5929-5936 (2012)

12. Borcut, M: Tripled coincidence theorems for contractive type mappings in partially ordered metric spaces. Appl. Math. Comput. 218, 7339-7346 (2012)

13. Karapınar, E, Luong, NV: Quadruple fixed point theorems for nonlinear contractions. Comput. Math. Appl. 64 $1839-1848(2012)$

14. Liu, X: Common fixed points of ordered g-contractions in partially ordered metric spaces. Fixed Point Theory Appl. (2014). doi:10.1186/1687-1812-2014-28

15. Kim, J, Chandok, S: Coupled common fixed point theorems for generalized nonlinear contraction mappings with the mixed monotone property in partially ordered metric spaces. Fixed Point Theory Appl. (2013). doi:10.1186/1687-1812-2013-307

16. Chandok, S, Karapinar, E, Khan, MS: Existence and uniqueness of common coupled fixed point results via auxiliary functions. Bull. Iran. Math. Soc. 40(1), 199-215 (2014)

17. Roldán, A, Martínez-Moreno, J, Roldán, C, Cho, YJ: Multidimensional coincidence point results for compatible mappings in partially ordered fuzzy metric spaces. Fuzzy Sets Syst. 251, 71-82 (2014)

18. Rad, GS, Shukla, S, Rahimi, H: Some relations between $n$-tuple fixed point and fixed point results. Rev. R. Acad. Cienc. Exactas Fís. Nat., Ser. A Mat. (2015). doi:10.1007/s13398-014-0196-0

19. Roldán, A, Martínez-Moreno, J, Roldán, C, Karapınar, E: Some remarks on multidimensional fixed point theorems. Fixed Point Theory 15, 545-558 (2014)

20. Samet, B, Karapınar, E, Aydi, H, Rajic, V: Discussion on some coupled fixed point theorems. Fixed Point Theory Appl. 2013, Article ID 50 (2013). doi:10.1186/1687-1812-2013-50

21. Chandok, S, Tas, K: An original coupled coincidence point result for a pair of mappings without MMP. J. Inequal. Appl. (2014). doi:10.1186/1029-242X-2014-61

22. Roldán, A, Martínez-Moreno, J, Roldán, C: Multidimensional fixed point theorems in partially ordered metric spaces. J. Math. Anal. Appl. 396, 536-545 (2012)

23. Berzig, M, Samet, B: An extension of coupled fixed point's concept in higher dimension and applications. Comput. Math. Appl. 63, 1319-1334 (2012)

24. Harjani, J, Sadarangani, K: Generalized contractions in partially ordered metric spaces and applications to ordinary differential equations. Nonlinear Anal. 72(3-4), 1188-1197 (2010)

25. Karapınar, E, Roldán, A, Martínez-Moreno, J, Roldán, C: Meir-Keeler type multidimensional fixed point theorems in partially ordered metric spaces. Abstr. Appl. Anal. 2013, Article ID 406026 (2013)

26. Wang, S: Multidimensional fixed point theorems for isotone mappings in partially ordered metric spaces. Fixed Point Theory Appl. 2014, Article ID 137 (2014). doi:10.1186/1687-1812-2014-137

27. Wang, S: Coincidence point theorems for G-isotone mappings in partially ordered metric spaces. Fixed Point Theory Appl. 2013, Article ID 96 (2013). doi:10.1186/1687-1812-2013-96

28. Ansari, $\mathrm{AH}$ : Note on ' $\varphi$ - $\psi$-contractive type mappings and related fixed point'. In: The 2 nd Regional Conference on Mathematics And Applications, PNU, September, pp. 377-380 (2014) 
29. Khan, MS, Swaleh, M, Sessa, S: Fixed point theorems by altering distances between the points. Bull. Aust. Math. Soc. 30, 1-9 (1984)

30. Babu, GVR, Sailaja, PD: A fixed point theorem of generalized weakly contractive maps in orbitally complete metric spaces. Thai J. Math. 9, 1-10 (2011)

31. Nieto, JJ, Pouso, RL, Rodríguez-Lopez, R: Fixed point theorems in partially ordered sets. Proc. Am. Math. Soc. 132(8), 2505-2517 (2007)

Submit your manuscript to a SpringerOpen ${ }^{\circ}$ journal and benefit from:

- Convenient online submission

Rigorous peer review

- Immediate publication on acceptance

- Open access: articles freely available online

- High visibility within the field

- Retaining the copyright to your article

Submit your next manuscript at $\boldsymbol{s p r i n g e r o p e n . c o m ~}$ 